\title{
Assessment of chromosomal rearrangements helps to differentiate multiple lung primary cancers from metastases
}

\author{
Laura Bonanno ${ }^{1}$, Alberto Pavan ${ }^{1,2}$, Stefano Indraccolo ${ }^{3}$ \\ ${ }^{1}$ Medical Oncology 2 Unit, Istituto Oncologico Veneto IOV IRCCS, Padova, Italy; ${ }^{2}$ Department of Surgery, Oncology and Gastroenterology, \\ Università degli Studi di Padova, Padova, Italy; ${ }^{3}$ Immunology and Molecular Oncology Unit, Istituto Oncologico Veneto IOV IRCCS, Padova, Italy \\ Correspondence to: Stefano Indraccolo, MD. Basic and Translational Oncology Unit, Istituto Oncologico Veneto IOV-IRCCS, via Gattamelata 64, \\ 35128 Padova, Italy. Email: stefano.indraccolo@unipd.it. \\ Provenance: This is an invited article commissioned by the Academic Editor Jingping Wang (Department of Pathology, The First Affiliated Hospital \\ of Bengbu Medical College, Bengbu Medical College, Bengbu, China). \\ Comment on: Murphy SJ, Harris FR, Kosari F, et al. Using Genomics to Differentiate Multiple Primaries From Metastatic Lung Cancer. J Thorac \\ Oncol 2019;14:1567-82.
}

Submitted Oct 10, 2019. Accepted for publication Nov 04, 2019.

doi: $10.21037 /$ tlcr.2019.11.09

View this article at: http://dx.doi.org/10.21037/tlcr.2019.11.09

During the last decades, genetic characterization of tumors has gained an increasing role in the development of new systemic treatments and one of the most commonly recognized needs in modern oncology is to use molecular characterization to tailor therapy. In many malignancies, but especially in non-small cell lung cancer (NSCLC), therapeutic algorithms are shaped based on genetic characterization and identification of driver alterations, which can be targeted with highly specific drugs (1). The current paradigm in precision oncology is that the more accurate we are in defining the disease, the best treatment we can administer to the patient in terms of efficacy, duration of clinical benefit and survival. This common ground is slowly but firmly pushing techniques for broad genetic characterization into daily practice of NSCLC. Next generation sequencing (NGS) allows screening of a large number of genetic alterations simultaneously and advanced stage disease is the first setting in which such analyses are currently applied.

Broad genetic characterization has also potential applications in early stage disease for prognostic definition and treatment customization in different clinical settings. Up to $8-15 \%$ of NSCLCs are diagnosed with multiple lung lesions and the differentiation of independent tumors from lung metastasis is a critical issue in their clinical management (2-4). The study of multiple lung nodules, especially when the disease is radiologically confined in the thorax, represents one potential field where genetic characterization might influence the multidisciplinary team's decision, which can range from the potentially curative to the palliative intent. As shown by multiple studies, the correct categorization of patients with multiple intrapulmonary cancer lesions is associated with improvement in outcome $(5,6)$. The first aim of pathological and molecular characterization is to distinguish between independent synchronous lung tumors and lung metastases, but it might also help in optimizing treatment of multifocal nodules of adenocarcinoma. For example, multiple nodules with ground glass features might benefit from loco-regional treatments (7).

Recently proposed criteria to distinguish metastatic foci from synchronous tumors still leave the issue fairly open, as the gold standard remains histological comparison (8). Histological assessment is based on histotype definition, description of architectural patterns, cytological features (cell size, nuclear and nucleolar features, amount of cytoplasm and quantity of mucin), tumor stroma and expression of thyroid transcription factor 1 (TTF1). The evaluation may be simple when tumor types are unequivocally different, but when histologic subtypes are similar, pathological review relies mainly on the skills and expertise of the pathologist, leading to an irreducible bias (9). In fact, inter-observer agreement rate on histopathological definition in resected specimens spans between $67 \%$ and 
$89 \%$ (10), whereas in biopsy specimens reaches $81 \%$ (11). Pathological definition is often integrated with clinical and radiologic information, being even more affected by interobserver variations. Defining cancer cell lineage through molecular characterization is consequently the cornerstone in order to overcome such limitations. Characterization of commonly mutated genes using standard genetic testing represents a first step in this direction.

In our experience, molecular profiling was useful in the treatment of a patient who had extra-thoracic cancer relapse, after radical surgery on three synchronous separate bilateral lung lesions (12). All three nodules were lung adenocarcinoma with a component of lepidic growth. Standard EGFR testing was able to define these nodules as three primary lung tumors, given the fact that one nodule carried an EGFR exon 19 deletion, the second carried an EGFR exon 21 classic mutation and the last one was EGFR wild type. The same test was also performed on the biopsy of the site of relapse and this permitted to establish the origin of metastatic diffusion and to choose systemic treatment accordingly (12).

Surely, in this context the less common is the mutation detected, the higher the probability of drawing clear conclusions, but several cases remain inconclusive, as genetic alterations might just identify a shared field of carcinogenesis (13). Moreover, different studies reported a substantial rate of discordance (from $12 \%$ to $45 \%$ ) between primary and metastatic sites of lung cancer for the most common driver mutations, such as EGFR mutations (14), and many cases may not show any of these alterations (15).

Genetic characterization through NGS is thus a promising tool to differentiate multiple primary lung tumors from pulmonary metastases: different lesions harboring the same mutational profile presumably derive from the same clone and are therefore classified as metastatic. In a large study that included 120 cases with multiple lung lesions, NGS analysis using panels covering over 500 hotspot mutations of $>20$ different genes was able to reduce the rate of inconclusive molecular classification to $9 \%$ and to define the correct lineage for the cases that were discordant at standard histological evaluation (16). In contrast, a smaller study (50 patients included), where a wider NGS panel of 50 genes was used to differentiate between multiple primary lung cancers and pulmonary metastases, could not improve the number of inconclusive evaluations, which remained frankly inconclusive in $2 \%$ and borderline non-conclusive in $22 \%$ of cases (17).

Murphy and colleagues in their recently published article (18) found that this rate of inconclusive NGS evaluations $(16,17)$ could be further reduced by mate-pair sequencing. This is a particular NGS analysis that has the potential to define lineage based on the discordance of the reading related to specific genetic alterations linked to the process of tumoral transformation $(18,19)$. The event of sharing the same somatic breakpoints and not having the same cell lineage has a very low probability to happen. This envisioned use of NGS is surely fascinating: the aim here is not the identification of driver genes involved in cancer development, but a genetic profiling based on the unique nature of chromosomal rearrangements in cancer, which represent a sort of genetic fingerprint of the tumor. In this study a total of 76 distinct fresh frozen tumor samples from 37 patients were analyzed, with 41 multiple comparisons performed between samples from the same patient. The cohort of tumors had already been evaluated by two pulmonary pathologists, blinded to molecular data. According to pathological analysis, $17 \%$ of pair comparisons were considered as indeterminate and $5 \%$ contrasted with molecular results. Notably, no case was deemed as not evaluable and no genomic analysis by mate-pair NGS had an indeterminate result (18). Overall, the method is innovative and has the potential to add information with respect to currently available tools, when integrated with pathological and clinical evaluation.

The main issues that intrinsically afflict this new declination of NGS regard its feasibility. The first point concerns the availability of adequate material for this type of analysis. Murphy and colleagues performed mate-pair NGS on surgically resected tissues, whereas most of daily oncological practice relies on diagnostics made on small biopsies or cell-blocks, which are not evaluable in $20 \%$ to $30 \%$ of cases (20). Tissue re-biopsy is not always feasible and its timing is not always consistent with clinical needs. This is surely a crucial issue, since the proposed method has a practical clinical application in the therapeutic perspective of patients. Moreover, this work retrospectively selected patients based on the availability of tissue of at least two distinct lung tumor sites: there are no data about the number of cases where this criteria was not met (18).

Secondly, an analysis that includes NGS-based testing of both tumor cell DNA and of blood-derived germline DNA, in order to filter results against germline structural genomic variations, is unavoidably expensive and time-consuming. These features are difficult to comply with a sustainable cancer care and with real world clinical practice (21).

A possible alternative strategy at diagnosis may involve 
the integration of other methods, such as medical artificial intelligence (AI), which is currently being developed based on computer science and big data deep learning. An AIbased diagnosis evaluation of radiological images of separate lung nodules was able to correctly identify pre-invasive and invasive lesions in a 53-patients-cohort of synchronous and metachronous multiple pulmonary nodules, with a rate of agreement with pathological evaluation of about $90 \%$ (22).

Future perspectives include the integration of different methods and professional competences: pathology, genetic analysis, radiology/radiomics and biostatistics. Although currently the differentiation of multiple primaries from metastatic lung cancer is still limited to the diagnostic step, in our opinion these algorithms will be increasingly integrated within multidisciplinary teamwork, which is essential to select and prescribe the best therapeutic program.

\section{Acknowledgments}

None.

\section{Footnote}

Conflicts of Interest: The authors have no conflicts of interest to declare.

Ethical Statement: The authors are accountable for all aspects of the work in ensuring that questions related to the accuracy or integrity of any part of the work are appropriately investigated and resolved.

\section{References}

1. Planchard D, Popat S, Kerr K, et al. Metastatic non-small cell lung cancer: ESMO Clinical Practice Guidelines for diagnosis, treatment and follow-up. Ann Oncol 2018;29:iv192-237.

2. Martini N, Malamed MR. Multiple primary lung cancers. J Thorac Cardiovasc Surg 1975;70:606-12.

3. Gazdar AF, Minna JD. Multifocal lung cancers--clonality vs field cancerization and does it matter? J Natl Cancer Inst 2009;101:541-3.

4. Girard N, Deshpande C, Lau C, et al. Comprehensive Histologic Assessment Helps to Differentiate Multiple Lung Primary Nonsmall Cell Carcinomas From Metastases. Am J Surg Pathol 2009;33:1752-64.

5. Jiang L, He J, Shi X, et al. Prognosis of synchronous and metachronous multiple primary lung cancers: Systematic review and meta-analysis. Lung Cancer 2015;87:303-10.

6. Chen K, Chen W, Cai J, et al. Favorable prognosis and high discrepancy of genetic features in surgical patients with multiple primary lung cancers. J Thorac Cardiovasc Surg 2018;155:371-9.e1.

7. Gu B, Burt BM, Merritt RE, et al. A Dominant Adenocarcinoma With Multifocal Ground Glass Lesions Does Not Behave as Advanced Disease. Ann Thorac Surg 2013;96:411-8.

8. Detterbeck FC, Franklin WA, Nicholson AG, et al. The IASLC Lung Cancer Staging Project: Background Data and Proposed Criteria to Distinguish Separate Primary Lung Cancers from Metastatic Foci in Patients with Two Lung Tumors in the Forthcoming Eighth Edition of the TNM Classification for Lung Cancer. J Thorac Oncol 2016;11:651-65.

9. Nicholson AG, Torkko K, Viola P, et al. Interobserver Variation among Pathologists and Refinement of Criteria in Distinguishing Separate Primary Tumors from Intrapulmonary Metastases in Lung. J Thorac Oncol 2018;13:205-17.

10. Paech DC, Weston AR, Pavlakis N, et al. A Systematic Review of the Interobserver Variability for Histology in the Differentiation between Squamous and Nonsquamous Non-small Cell Lung Cancer. J Thorac Oncol 2011;6:55-63.

11. Yamamoto S, Sobue T, Yamaguchi N, et al. Reproducibility of Diagnosis and Its Influence on the Distribution of Lung Cancer by Histologic Type in Osaka, Japan. Jpn J Cancer Res 2000;91:1-8.

12. Bonanno L, Calabrese F, Nardo G, et al. Morphological and genetic heterogeneity in multifocal lung adenocarcinoma: The case of a never-smoker woman. Lung Cancer 2016;96:52-5.

13. Curtius K, Wright NA, Graham TA. An evolutionary perspective on field cancerization. Nat Rev Cancer 2018;18:19-32.

14. Vignot S, Besse B, André F, et al. Discrepancies between primary tumor and metastasis: A literature review on clinically established biomarkers. Crit Rev Oncol Hematol 2012;84:301-13.

15. Asmar R, Sonett JR, Singh G, et al. Use of Oncogenic Driver Mutations in Staging of Multiple Primary Lung Carcinomas: A Single-Center Experience. J Thorac Oncol 2017;12:1524-35.

16. Mansuet-Lupo A, Barritault M, Alifano M, et al. Proposal 
for a Combined Histomolecular Algorithm to Distinguish Multiple Primary Adenocarcinomas from Intrapulmonary Metastasis in Patients with Multiple Lung Tumors. J Thorac Oncol 2019;14:844-56.

17. Roepman P, Ten Heuvel A, Scheidel KC, et al. Added Value of 50-Gene Panel Sequencing to Distinguish Multiple Primary Lung Cancers from Pulmonary Metastases. J Mol Diagn 2018;20:436-45.

18. Murphy SJ, Harris FR, Kosari F, et al. Using Genomics to Differentiate Multiple Primaries From Metastatic Lung Cancer. J Thorac Oncol 2019;14:1567-82.

19. Murphy SJ, Cheville JC, Zarei S, et al. Mate Pair Sequencing of Whole-Genome-Amplified DNA Following
Laser Capture Microdissection of Prostate Cancer. DNA Res 2012;19:395-406.

20. Vanderlaan PA, Yamaguchi N, Folch E, et al. Success and failure rates of tumor genotyping techniques in routine pathological samples with non-small-cell lung cancer. Lung Cancer 2014;84:39-44.

21. Tan O, Shrestha R, Cunich M, et al. Application of nextgeneration sequencing to improve cancer management: A review of the clinical effectiveness and cost-effectiveness. Clin Genet 2018;93:533-44.

22. Li X, Hu B, Li H, et al. Application of artificial intelligence in the diagnosis of multiple primary lung cancer. Thorac Cancer 2019;10:2168-74.
Cite this article as: Bonanno L, Pavan A, Indraccolo S. Assessment of chromosomal rearrangements helps to differentiate multiple lung primary cancers from metastases. Transl Lung Cancer Res 2019;8(Suppl 4):S435-S438. doi: 10.21037/tlcr.2019.11.09 